\title{
EFECTO ANTITROMBÓTICO, UNA CARACTERÍSTICA POCO CONOCIDA DE LAS FRUTAS Y HORTALIZAS.
}

\section{ANTITHROMBOTIC EFFECT, A NOT WELL KNOWN CHARACTERISTIC OF FRUITS AND VEGETABLES}

\author{
Constanza Torres U., Luís Guzmán J., Rodrigo Moore-Carrasco., \\ Iván Palomo G.
}

Programa de Investigación en Factores de Riesgo de Enfermedad Cardiovascular (PIFRECV)

Departamento de Bioquímica Clínica e Inmunohematología, Facultad de Ciencias de la Salud, Universidad de Talca.

Este trabajo fue recibido el 2 de Enero de 2008 y aceptado para ser publicado el 3 de Marzo de 2008.

Dirigir la correspondencia a:

Sr.

Iván Palomo G., PhD.

Departamento de Bioquímica Clínica e Inmunohematología

Facultad Ciencias de la Salud

Universidad de Talca

Talca, Chile.

P.O. box: 747, Talca, Chile

E-mail: ipalomo@utalca.cl

Fone: 56-71-200493

Fax : 56-71-200488 


\section{ABSTRACT}

Cardiovascular diseases (CVD) are the leading cause of death in the world. Several risk factors for CVD, such as lipid disorders, hypertension and diabetes mellitus, are influenced by food. It is well known that fruits and vegetables contain antioxidants and it adequate consumption reduces cardiovascular risk. However, its antithrombotic effect (antiplatelet agent, anticoagulant and fibrinolytic) is little known. This review briefly describes these effects, both in vivo and in vitro, and the possible mechanisms that could explain this effect. Fruits such as black grape, pineapple, strawberry and kiwi show this effect. Among the vegetables that have antiaggregatory effect are garlic, onions, welsh onions, tomatoes and melons. On the other hand the anticoagulant effect has only been found in fruits like pineapple, and among the vegetables in garlic and onions. The fibrinolytic effect has been described in fruits like kiwi and pineapple, an in vegetables such as garlic, onions and soybeans. Some fruits (pineapple and kiwi) and vegetables (onion and garlic) have more than one antithrombotic effect so their regular consumption certainly protects from CVD. We have begun the study, initially in vitro of the potential antithrombotic effect of fruits and vegetables in the Maule Region. It is necessary to increase our domestic consumption and export of fruits and vegetables, both to improve the health of the population and the economy. The reasons above stated describe the importance of the contribution of knowledge due to the fact that antioxidant effects are less known.

Keywords: Fruits, vegetables, antiaggregant, anticoagulant, fibrinolytic. 


\section{INTRODUCCIÓN}

Las enfermedades cardiovasculares (ECV) lideran las causas de muerte en el mundo, especialmente en los países desarrollados y en vías de desarrollo [1]. Varios de los factores de riesgo de las ECV, incluyendo dislipidemias, hipertensión arterial y diabetes mellitus, en parte importante son influenciados por la alimentación [2, 3].

La Encuesta Nacional de Salud, 2003, mostró que un 50\% de la población adulta (18-74 años) chilena presentó riesgo de ECV [4]. En Talca el 44,5\% de los adultos presentó hipercolesterolemia, $40,1 \%$ hipertrigliceridemia y $73,4 \%$ obesidad y sobrepeso [5], todos factores de riesgo cardiovascular.

Evidencias epidemiológicas indican que una dieta rica en frutas y hortalizas promueve la salud, disminuyendo el riesgo a desarrollar ECVs y otras enfermedades crónicas [6]. El aporte en antioxidantes de las frutas y hortalizas es bastamente reconocido [7, 8], sin embargo su efecto antitrombótico es menos conocido [9].

Para promover el consumo nacional de frutas y verduras las Asociaciones Internacionales [2], los Ministerios de Salud y de Agricultura de Chile [10], y el Instituto Nacional de Tecnología de los Alimentos (INTA) iniciaron durante 2007 la campaña "5 al día", que promueve el consumo de cinco o más frutas y verduras diarias [10,11]. Por otra parte, el Gobierno de Chile se ha planteado la meta de transformar al país en una potencia agroalimentaria para el Bicentenario $[10,12]$. Conseguir un aumento del consumo interno y de las exportaciones, requiere de una estrategia en que participen todos los actores involucrados, y en este contexto parece necesario que grupos dedicados a la salud cardiovascular investiguen, además de los efectos antioxidantes de las frutas y verduras, sobre sus efectos antitrombóticos especialmente en las regiones del país cuya principal actividad económica es la agrícola, como son las VI y VII. Dichos estudios deberían incluir ensayos in vitro, experimentos en animales de experimentación y estudios en humanos, en todos los cuales se investiguen diferentes especies y variedades, según territorios y otras variables.

A continuación se describirán los efectos antitrombóticos (antiagregante plaquetario, anticoagulante y fibrinolítico) descritos en frutas y verduras (tabla 1):

\section{Tabla 1}

Frutas y hortalizas que presentan algún tipo de efecto antitrombótico

\begin{tabular}{|c|c|c|}
\hline \multirow{3}{*}{ Efecto antiagregante plaquetario } & Frutas & Hortalizas \\
\hline & Uva negra & Ajo \\
\hline & $\begin{array}{l}\text { Frutilla } \\
\text { Kiwi } \\
\text { Piña }\end{array}$ & $\begin{array}{l}\text { Cebolla } \\
\text { Cebollín } \\
\text { Tomate } \\
\text { Melón }\end{array}$ \\
\hline Efecto anticoagulante & Piña & $\begin{array}{l}\text { Ajo } \\
\text { Cebolla }\end{array}$ \\
\hline Efecto fibrinolítico & $\begin{array}{l}\text { Kiwi } \\
\text { Piña }\end{array}$ & $\begin{array}{l}\text { Ajo } \\
\text { Cebolla } \\
\text { Soya }\end{array}$ \\
\hline
\end{tabular}




\section{EFECTO ANTIAGREGANTE PLAQUETARIO DE FRUTAS Y HORTALIZAS}

\section{Frutas}

El efecto antiagregante plaquetario se ha observado en varias frutas: uva negra (Vitis vinifera), piña (Ananas sativus), frutilla (Fresalfragaria vesca) y kiwi (Actinidia deliciosa).

Uva negra. El consumo diario de jugo de uva negra $(5-7.5 \mathrm{ml} / \mathrm{kg} /$ día $)$, en personas adultas (26-58 años) durante 7-10 días redujo la agregación plaquetaria inducida por colágeno $(1 \mathrm{mg} / \mathrm{l})$ en un $77 \%$ [13]. El mecanismo por el cual el jugo de uva negra inhibe la actividad plaquetaria no es bien conocido, pero se ha planteado que bs flavonoides (flavonoles, antocyanidinas y proantocianidinas) de uvas inhiben las enzimas ciclooxigenasa y fosfodiesterasa $[14,15]$. Por otra parte, el jugo de uva negra y el vino tinto aumentan la producción de óxido nítrico (NO), tanto plaquetaria como endotelial [16]. Otro estudio mostró, en ensayos in vitro, que el jugo de uva y flavonoides derivados de la uva negra inhiben la agregación plaquetaria, aumentan la liberación de NO derivado de plaquetas y disminuyen la producción de superóxido; dicho efecto fue confirmado en veinte individuos sanos que tomaron $7 \mathrm{ml} / \mathrm{kg} /$ día de jugo de uva por 14 días [17].

Frutilla. Los compuestos activos más abundantes en las frutillas son el ácido elágico y ciertos flavonoides como antocianina, catequina, quercitina y kaempferol, los que tienen propiedades antioxidantes [18]. Filtrados de tres variedades de frutillas, KYSt-4 (Nohime), KYSt-11 (Kurume IH-1) y KYSt-17 (Kurume 58), han mostrado un significativo efecto antiplaquetario in vitro y en ratones [19]. En el mismo estudio se encontró una correlación significativa entre la actividad antioxidante y antiplaquetaria de los compuestos fenólicos; además este último efecto era estable al calor [19]. Se ha observado que los extractos de frutillas inhiben in vitro algunas enzimas como la ciclooxigenasa [18] lo que podría explicar en parte el efecto antiagregante plaquetario.

Kiwi. En estudios de agregación plaquetaria in vitro inducida por adenosindifosfato (ADP) se ha observado inhibición dosis dependiente (con $5 \mathrm{mg}$ de extracto de kiwi 11\% de inhibición, $10 \mathrm{mg}$ de extracto 54\% de inhibición y $20 \mathrm{mg}$ de extracto $96 \%$ de inhibición). El extracto también inhibió la agregación con colágeno, pero los niveles fueron mucho menores con 5 y $10 \mathrm{mg}$. Sin embargo con ácido araquidónico, como agonista, el porcentaje de inhibición de la agregación plaquetaria fue de sólo un 32\%, con $20 \mathrm{mg}$ de extracto. Estos resultados sugieren que la vía del tromboxano no parece estar involucrada y se necesitan más estudios para determinar la estructura y mecanismo de acción responsables de estos efectos. [20]. También se evaluó si el consumo de kiwi modulaba la actividad plaquetaria en un grupo (randomizado) de 30 personas (20-51 años) voluntarias. Se observó que la respuesta plaquetaria a los 28 días, a altas y bajas concentraciones de ADP, fue inhibida significativamente por el consumo de 2 kiwis/día (18\% en el caso de ADP $4 \mu \mathrm{M}$ y $15 \%$ con ADP 8 $\mu \mathrm{M}$, comparados con el día 0). También se observó una reducción similar en la agregación plaquetaria en respuesta a colágeno, cuando los voluntarios consumieron 2 o 3 kiwis/día durante 28 días. Estos antecedentes indican que el consumo de kiwis podría prevenir el desarrollo de eventos trombóticos [20].

Piña. El extracto acuoso y crudo de los tallos y frutos inmaduros de la piña, denominado extracto de bromelina, constituye una compleja mezcla de diferentes endopeptidasas-tiol y otros componentes aún no completamente caracterizados tales como fosfatasas, glucosidasas, peroxidasas, glicoproteínas y carbohidratos [21]. En un estudio se investigó el efecto de la bromelina sobre la agregación plaquetaria in vitro, encontrándose que a una concentración de $2.5 \mathrm{mg} / \mathrm{ml}$ inhibe la

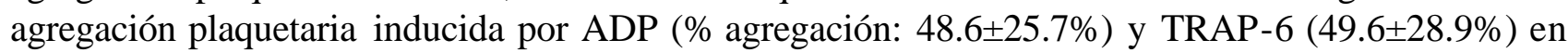

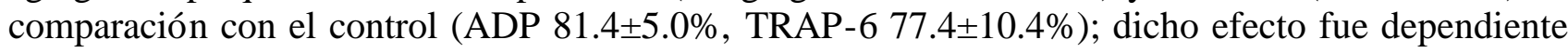


de concentración [22]. Además, usando citometría de flujo, se determinó que el porcentaje de plaquetas no estimuladas CD62P(+) (\%PP), fue mínimamente mayor luego de la incubación con 5 $\mathrm{mg} / \mathrm{ml}$ de bromelina $(0.57 \pm 0.48 \% \mathrm{PP})$ comparado con el control $(0.22 \pm 0.11 \% \mathrm{PP})$, pero luego de la estimulación con TRAP-6, la incubación con $5 \mathrm{mg} / \mathrm{ml}$ de bromelina, llevó a una notoria disminución en comparación con el control no tratado $(50.4 \pm 20.2$ a $0.9 \pm 0.8 \% \mathrm{PP})$ [22].

\section{HORTALIZAS}

Las hortalizas a las que se ha descrito efecto antiagregante plaquetario son el ajo (Allium sativum), la cebolla (Allium cepa), el cebollín (Allium schoenoprasum), el tomate (Lycopersicon esculentum) y el melón (Cucurbitacea cucumis melo).

Ajo y cebolla. Estos miembros de la familia Allium, han sido utilizados desde hace muchos años como alimento con propiedades medicinales para tratar una variedad de enfermedades, entre otras, las enfermedades cardíacas [23]. Lo anterior se ha explicado fundamentalmente por su efecto antiagregante plaquetario e hipotensor [24].

El consumo por largo tiempo de ajo crudo, ajo en polvo, aceite de ajo y extractos de ajo envejecidos inhiben la agregación plaquetaria en humanos [25-28]. Estudios in vitro del efecto de extractos acuosos, crudos y hervidos de ajo y cebolla, sobre la agregación plaquetaria inducida por colágeno, han mostrado diferentes grados de inhibición usando plasma rico en plaquetas de conejo (IC50 Ajo $8.8 \mathrm{mg} / \mathrm{ml}$ ) y de humanos (IC50 Ajo 6,6 mg/ml, IC50 Cebolla 90 mg/ml). Sólo la inhibición causada por el ajo en plaquetas humanas fue dosis dependiente. Los extractos hervidos mostraron un leve efecto inhibitorio [29]. Las propiedades antitrombóticas del ajo y la cebolla han sido atribuidas a compuestos organosulfurados formados ślo cuando estas hortalizas son trituradas y la enzima allinasa es liberada al medio [30-32]; los efectos antiplaquetarios in vivo de la ingestión del ajo son atribuidos nás a la adenosina que a los constituyentes polisulfidos [33]. Los tiosulfinatos son el mayor compuesto sulfidrilo producido en especies Allium recién cortadas [34]. Allicin (allyl 2propenethiosulfinate) es formado desde su precursor alliin (S-2- propenyl-L-cysteine sulfoxide), siendo el tiosulfinato predominante en el ajo [35] y un potente inhibidor plaquetario [36].

Cebollín. Esta hortaliza también es miembro de la familia Allium. El cebollín es un importante aderezo vegetal, en las comidas asiáticas [37] y se le ha consumido para prevenir las ECV; sin embargo, su mecanismo es aún desconocido [38]. La administración exógena de extractos de cebollín, especialmente la porción verde, cruda, parece tener efectos inhibitorios de la adhesión y agregación de plaquetas humanas in vitro usando ADP como agonista [38]. En ratas SpragueDawley $\mathbf{e}$ evaluó el efecto antitrombótico administrándoles intragástricamente jugo de cebollín crudo (2 gr/kg/día, durante 28 días), en comparación con ratas que se les administró jugo de cebollín hervido y con un grupo control; las ratas tratadas con jugo crudo mostraron prolongación del tiempo de sangría, y disminución de la adhesión plaquetaria a superficies cubiertas con fibrinógeno, de la agregación plaquetaria con ADP y de la liberación de tromboxano inducidas por ADP; además se observó elevación de la concentración de AMPc de las plaquetas [37]. En humanos también se ha descrito efecto antiplaquetario del extracto del cebollín, crudo pero no hervido [38].

Tomate. El jugo de tomate (20-50 $\mu \mathrm{l})$ también han mostrado actividad antiplaquetaria in vitro al inhibir la agregación inducida por ADP y colágeno sobre un 70\% [39]. No todas la variedades de tomates presentan efecto antiagregante; la variedad KG99-4 mostró un potente efecto antitrombótico in vitro utilizando un hemostatómetro (ratas Wistar ST) y también luego de su administración oral inhibió la trombosis arterial (trombos ricos en plaquetas) inducida por láser (ratones C57BL/6, dosis de $3.85 \mathrm{ml} / \mathrm{kg}$ en 2 administraciones: al inicio y a los 30 minutos, los ensayos comenzaron luego de 90 minutos desde la segunda administración) [40]. 
En un estudio randomizado, doble ciego, placebo controlado cruzado, a 90 adultos (45-70 años) aparentemente sanos, con función plaquetaria normal, se les evaluó el efecto del consumo de tomates. Se observaron significativas reducciones en la agregación plaquetaria luego de 3 horas de haber consumido extractos equivalentes a 2 y 6 tomates, respectivamente: $-12.7 \%,-21.3 \%$ (ADP 3 $\mu \mathrm{M}),-7.6 \%,-7.8 \%$ (ADP $7.5 \mu \mathrm{M}$ ) y $-14.6 \%,-17.5 \%$ (colágeno) [41]. La inhibición fue mayor en los hombres que en las mujeres [41].

En otro estudio durante 7 horas, a 23 personas aparentemente sanas (40- 65 años) canuladas, se les realizó varias mediciones para determinar la eficacia y la duración de los efectos antiplaquetarios de un suplemento que contenía extracto de tomate. A las 3 horas posteriores a una dosis equivalente a 6 tomates, se reportó una inhibición significativa de la función plaquetaria, con respecto a la medición basal, de $2.9 \pm 1.4 \%$ (ADP $8 \mu \mathrm{M}$ ) y de $20.0 \pm 4.9 \%$ (ADP $3 \mu \mathrm{M}$ ). Los efectos observados persistieron por más de 12 horas [42].

Se ha sugerido que el antioxidante licopeno, presente en alta concentración en los tomates, y componentes estables al calor y de bajo peso molecular como la adenosina y otros, podrían ser responsables del efecto antiplaquetario [39, 43]. Durante el proceso de maduración del tomate aumenta la concentración de licopenos y disminuye la actividad antiplaquetaria [39]; esto resta fuerza a la participación de los licopenos en el citado efecto. Lo anterior podría significar que el diferente potencial antitrombótico observado en distintas variedades de tomates, se explique por la existencia de más de un componente activo y/o diferente concentración de los mismos [40].

El extracto de tomate como suplemento dietario o alimento funcional, puede tener un rol en la prevención primaria de las enfermedades cardiovasculares reduciendo la activación plaquetaria, lo cual puede contribuir a reducir de los efectos trombóticos.

Melón. Otra hortaliza en la que se ha encontrado acción antiagregante es el melón [44]. Una fracción de extracto acuoso del melón no identificada inhibió la agregación plaquetaria inducida por epinefrina, ADP, colágeno, trombina, ácido araquidónico, factor activador de plaquetas (PAF) y la molécula U-46619 [44].

\section{EFECTO ANTICOAGULANTE DE FRUTAS Y HORTALIZAS}

\section{Frutas}

Piña. La bromelina, una enzima proteolítica derivada del tallo y fruto de la piña por extracción acuosa, precipitación o secado posee actividad antitrombina, reportándose que su efecto in vitro

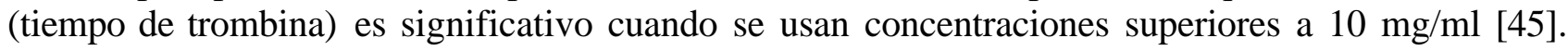
Este efecto es mayor cuando la bromelina y la trombina $(50 \mathrm{UI} / \mathrm{ml})$ se incuban juntas a $37^{\circ} \mathrm{C}$ [45].

\section{HORTALIZAS}

Ajos y cebollas. Las especies Allium, especialmente el ajo y la cebolla, contienen sustancias biológicamente activas con efecto anticoagulante. Aunque los componentes órgano-sulfurados presentes en las especies Allium han sido reportados como anticoagulantes, hay pocos reportes sobre el efecto antitrombótico de la cebolla [46].

Macerado de cebollas que fueron mantenidos por 30 minutos en buffer Tris-HCL a pH 7.0 mostró actividad anticoagulante (antitrombina), dosis-dependiente; dicho extracto mantuvo su actividad anticoagulante al ser sometido a $100^{\circ} \mathrm{C}$ por 30 minutos y a pH 2.0 por 4 horas, pero cuando el extracto se dializó con una membrana de celulosa perdió la actividad, sugiriendo que la(s) moléculas 
responsables serían de bajo peso molecular [47]. Por otra parte, no todas las variedades de cebollas presentan efecto anticoagulante; de diez variedades estudiadas (Toyohira, Tsukisappu, Rantaro, Kitamiko27, Kitawase3, Superkitamomiji, CS3-12, Tsukiko22, 2935A y K83211), sólo las tres primeras presentaron efectos anticoagulantes en un hemostatómetro [48]. El mecanismo por el cual ejercen su efecto anticoagulante aún no está claro y son necesarios más estudios para identificar los componentes que tienen dicha actividad.

\title{
EFECTO FIBRINOLÍTICO DE FRUTAS Y HORTALIZAS
}

\begin{abstract}
Frutas
Kiwi. Este fruto nativo del Asia Oriental una de las pocas frutas descritas con alguna propiedad fibrinolítica [49]. Los kiwis entre otros compuestos contienen vitamina C, carotenoides, flavonoides y minerales [50]. Se ha demostrado mediante la técnica de placa de fibrina de Astrup y Mullertz [51], que el kiwi posee actividad fibrinolítica a concentraciones entre 50 y $100 \mathrm{mg} / \mathrm{ml}$, tanto en extractos acuosos como en $70 \%$ etanol [52].
\end{abstract}

Piña. Es otra de las frutas que ha demostrado un efecto fibrinolítico, en este caso asociado a la bromelina de una familia de enzimas proteolíticas sulfidrílicas [22].

Varios estudios sobre los efectos de la bromelina en la circulación, atribuyen su utilidad a la activación del plasminógeno y el subsecuente incremento en la actividad fibrinolítica [53].

Se ha observado que la administración oral de bromelina a ratas Sprague-Dawley estimula la actividad fibrinolítica en el suero asociada a la activación del plasminógeno por la bromelina [5355].

\section{Hortalizas}

Ajo. Se ha observado que el ajo en polvo inodoro (odorless garlic powder) mejora el sistema fibrinolítico por el incremento de la actividad del activador del plasminógeno tipo tisular (t-PA) in vitro [56]. Por otra parte, se ha comunicado que el aceite de ajo o su polvo aumenta la actividad fibrinolítica en humanos, efecto que al parecer no estar mediado por el incremento en la secreción de t-PA o por la disminución en la secreción de inhibidor del activador del plasminógeno-1 (PAI-1) desde las células endoteliales, sino más bien por un aumento en la actividad de t-PA [56]. Incrementos en la generación de plasmina pueden observarse a bajas dosis de ajo (1, 10 y 100 $\mathrm{mg} / \mathrm{ml}$ ), pero un incremento dosis dependiente de la plasmina puede encontrarse bajo altas dosis de ajo (500 y $1000 \mathrm{mg} / \mathrm{ml})$. Estudios cinéticos han mostrado la disminución en la $\mathrm{Km}$ en presencia del ajo, sugiriendo que el ajo incrementa la afinidad entre el t-PA y el plasminógeno. Además, pareciera que los componentes activos del ajo podrían actuar como cofactores que hacen al t-PA más accesible al plasminógeno por exposición de sus sitios de afinidad para el plasminógeno más eficientemente [56].

Algunos estudios en humanos [57], han demostrado que la ingestión de ajo (10 gramos/día de ajo crudo por 2 meses), trae consigo una disminución en el colesterol sérico y un incremento en la actividad fibrinolítica en jóvenes (17 y 22 años) normales. La ingesta diaria de ajo en la dieta podría prevención la aparición de eventos trombóticos [58].

Cebolla. El consumo de cebollas promueve un aumento de la actividad fibrinolítica [59]. Además, se ha demostrado que distintas variedades de cebollas poseen distintos efectos antitrombóticos y trombolíticos tanto in vivo como in vitro [48]. 
Soya (Glycine max L). Con uno de sus productos derivados Natto, un vegetal fermentado como el queso el cual es una comida tradicional. Los extractos del Natto, incluyen la Natokinasa, una potente enzima fibrinolítica la cual posee aproximadamente cuatro veces mayor actividad que la plasmina en los ensayos de lísis del coágulo [60, 61].

Se ha encontrado que la Natokinasa acorta los tiempos de lisis del coágulo de euglobulina. Dado que el tiempo de lisis del coágulo de euglobulina expresa la total actividad fibrinolítica intrínseca del plasma, al parecer los extractos de Natto aumentan la actividad del t-PA [60].

\section{ESTUDIOS EN NUESTRO LABORATORIO}

Hemos iniciado el estudio, por el momento in vitro, del eventual efecto antitrombótico de frutas y hortalizas. Para ello, en el caso de las frutas, comenzamos a trabajar con extractos acuosos (jugo), alcohólicos (pulpa) y de las hortalizas, sólo en metanol. Inicialmente los extractos están siendo evaluados a $1 \mathrm{mg} / \mathrm{ml}$.

Las pruebas que estamos utilizando para evaluar los eventuales efectos antitrombóticos de las frutas y hortalizas (dos o tres concentraciones), son las siguientes:

Efecto antiagregante plaquetario: Agregación plaquetaria, inicialmente con dos agonistas (ADP y ácido araquidónico).

Efecto anticoagulante: Tiempo de protrombina, tiempo de tromboplastina parcial activado, tiempo de trombina, tiempo de protrombina diluido, tiempo de coagulación con kaolín y curva de generación de fibrina.

Efecto fibrinolítico: Placa de fibrina y prueba de lísis del coágulo de euglobulina.

\section{COMENTARIO FINAL}

En el contexto: (a) que las ECV son la principal causa de morbimortalidad y que son prevenibles focalizándose en los factores de riesgo modificables y sus condicionantes, (b) que las frutas y hortalizas protegen de las ECV, (c) que debemos hacer esfuerzos para aumentar el consumo interno y (d) que el país desea transformarse en potencia agroalimentaria, pasa a ser relevante toda contribución que se pueda hacer al conocimiento de los FRCV en su asociación con estrategias de intervención en que se priorice la participación de frutas y verduras. En ese sentido una de nuestras líneas de trabajo se refiere al estudio de los efectos antitrombóticos de frutas y vegetales en estudios in vitro y próximamente e in vivo (modelos murino y en humanos) [9].

Además de aumentar las exportaciones de frutas y hortalizas, las autoridades deben preocuparse por promover el desarrollo de los mercados internos para las frutas nacionales; el consumo a nivel local, debe fortalecerse a través de campañas de promoción que incorporen conceptos de alimentación saludable y vida sana relacionados a la ingesta de frutas; campañas fundamentadas en beneficios para la salud con información científica.

\section{RESUMEN}

Las enfermedades cardiovasculares (ECV) son la principal causa de mortalidad en el mundo. Varios de los factores de riesgo de las ECV, como dislipidemias, hipertensión arterial y diabetes mellitus, son influenciados por la alimentación. Es conocido que las frutas y hortalizas contienen antioxidantes, y que su consumo en una cantidad adecuada disminuye el riesgo cardiovascular. Sin embargo, su efecto antitrombótico (antiagregante plaquetario, anticoagulante y fibrinolítico) es poco conocido. En esta revisión se describen brevemente dichos efectos, tanto in vitro como in vivo, y los posibles mecanismos que podrían explicar éstos. En cuanto al efecto antiagregante plaquetario, entre las frutas que poseen dicha característica se incluyen uva negra, piña, futilla y kiwi. Entre las 
hortalizas en que se ha descrito efecto antiagregante están el ajo, la cebolla, el cebollín, el tomate y el melón. Por su parte, el efecto anticoagulante, entre las frutas, sólo se ha encontrado en la piña, y entre las hortalizas en ajos y cebollas. El efecto fibrinolítico se ha descrito en frutas como el kiwi y la piña, y hortalizas como el ajo, las cebollas y la soya. Algunas frutas (piña y kiwi) y hortalizas (ajo y cebollas) presentan más de un efecto antitrombótico por lo que seguramente su consumo regular protege de las ECV. Nosotros hemos iniciado el estudio, por lo pronto in vitro, del posible efecto antitrombótico de frutas y hortalizas de la Región del Maule. Siendo necesario aumentar el consumo interno y las exportaciones de frutas y hortalizas, tanto para mejorar la salud de la población como desde el punto de vista económico, parece relevante contribuir al conocimiento de los efectos aquí descritos, los que son menos conocidos que el efecto antioxidante.

Palabras claves: Frutas, hortalizas, antiagregante, anticoagulante, fibrinolítico.

Agradecimientos: Al Programa de Investigación en Factores de Riesgo de Enfermedades Cardiovasculares (PIFRECV) y la Dirección de Investigación de la Universidad de Talca.

\section{BIBLIOGRAFIA}

1.- $\quad$ WHO World Health Organization Informe sobre la salud en el mundo, in Technical Report Series. 2002: Geneve.

2.- $\quad$ Liu S. Manson J.E. Lee I.M. Cole S.R. Hennekens C.H. Willett W.C. Buring J.E. Fruit and vegetable intake and risk of cardiovascular disease: the Women's Health Study. Am J Clin Nutr 2000; 72(4): 922-928.

3.- $\quad$ De Vet E. de Nooijer J. de Vries N. K.Brug J. The Transtheoretical model for fruit, vegetable and fish consumption: associations between intakes, stages of change and stage transition determinants. Int J Behav Nutr Phys Act 2006; 3: 13.

4.- $\quad$ MINSAL Ministerio de Salud, Gobierno de Chile. Resultados $1^{a}$ Encuesta de Salud. 2003; Disponible en: http://epi.minsal.cl/epi/html/invest/ENS/InformeFinalENS.pdf

5.- $\quad$ Palomo I. Icaza G. Mujica V. Nuñez L. Leiva E. Vásquez M. Alarcón M. Moyano E. . Prevalencia de factores de riesgo cardiovascular clásicos en población adulta de Talca, Chile, 2005. Rev Méd Chile 2007; 135: 904-912.

6.- $\quad$ Rimm E.B. Fruit and vegetables--building a solid foundation. Am J Clin Nutr 2002; 76(1): 1-

2.

7.- $\quad$ Avello M. Suwalsky M. Radicales libres, antioxidantes naturales y mecanismos de protección. Atenea (Concepc.) 2006; 494: 161-172.

8.- De Angelis R.C. Novos conceitos em nutrição: reflexões a respeito do elo dieta e saúde. Arqu Gastroenterol 2001; 38: 269-271.

9.- $\quad$ Palomo I. Efectos antitrombóticos de las frutas y hortalizas, En Dieta Mediterránea. Prevención de las Enfermedades Cardiovasculares ed. Palomo I. Leiva E. Vásquez M. Editorial Universidad de Talca. 2007.

10.- $\quad$ MINAGRI Ministerio de Agricultura Una politica de Estado para la Agricultura Chilena, Periodo 2000 - 2010. Disponible en: http://www.minagri.gob.cl/politica/politic2000-2010.pdf. 11.- INTA Instituto de Tecnología en Alimentos. Programa 5 al Día en Chile. Disponible en: http://www.inta.cl/5aldia/5aldia.pdf.

12.- INTA Instituto de Tecnología en Alimentos. Reunión de coordinación para implementar el programa "5 al Día" en Chile. Disponible en: http://www.inta.cl/noticias/5aldia/reunion.asp.

13.- Keevil J.G. Osman H.E. Reed J.D. Folts J.D. Grape juice, but not orange juice or grapefruit juice, inhibits human platelet aggregation. J Nutr 2000; 130(1): 53-56. 
14.- Silva J. Riguld J. Cheynier V. Cheminat A. Moutounet M. Procyanidin dimers and trimers from grape seeds. Phytochemistry 1991; 30: 1259-1264.

15.- Laughton M.J. Evans P.J. Moroney M.A. Hoult J.R. Halliwell B. Inhibition of mammalian 5lipoxygenase and cyclo-oxygenase by flavonoids and phenolic dietary additives. Relationship to antioxidant activity and to iron ion-reducing ability. Biochem Pharmacol 1991; 42(9): 1673-1681.

16.- Folts J.D. Potential health benefits from the flavonoids in grape products on vascular disease. Adv Exp Med Biol 2002; 505: 95-111.

17.- $\quad$ Freedman J.E. Parker C. Li L. Perlman J.A. Frei B. Ivanov V. Deak L.R. Iafrati M.D. Folts

J.D. Select flavonoids and whole juice from purple grapes inhibit platelet function and enhance nitric oxide release. Circulation 2001; 103(23): 2792-2798.

18.- Hannum S.M. Potential impact of strawberries on human health: a review of the science. Crit Rev Food Sci Nutr 2004; 44(1): 1-17.

19.- Naemura A. Mitani T. Ijiri Y. Tamura Y. Yamashita T. Okimura M. Yamamoto J. Antithrombotic effect of strawberries. Blood Coagul Fibrinolys is 2005; 16(7): 501-509.

20.- Duttaroy A.K. Jørgensen A. Effects of kiwi fruit consumption on platelet aggregation and plasma lipids in healthy human volunteers. Platelets 2004; 15(5): 287 - 292.

21.- Maurer H.R. Bromelain: biochemistry, pharmacology and medical use. Cell Mol Life Sci 2001; 58(9): 1234-1245.

22.- Glaser D. Hilberg T. The influence of bromelain on platelet count and platelet activity in vitro. Platelets 2006; 17(1): 37-41.

23.- $\quad$ Fenwick G.R. Hanley A.B. The genus Allium-Part 1. Crit Rev Food Sci Nutr 1985; 22(3): 199-271.

24.- Rahman K. Historical perspective on garlic and cardiovascular disease. J Nutr 2001; 131(3s): 977S-979S.

25.- $\quad$ Bordia A. Sharma K.D. Parmar Y.K. Verma S.K. Protective effect of garlic oil on the changes produced by 3 weeks of fatty diet on serum cholesterol, serum triglycerides, fibrinolytic activity and platelet adhesiveness in man. Indian Heart J 1982; 34(2): 86-88.

26.- $\quad$ Bordia T. Mohammed N. Thomson M. Ali M. An evaluation of garlic and onion as antithrombotic agents. Prostaglandins Leukot Essent Fatty Acids 1996; 54(3): 183-186.

27.- Cavagnaro P.F. Camargo A. Galmarini C.R. Simon P.W. Effect of cooking on garlic (Allium sativum L.) antiplatelet activity and thiosulfinates content. J Agric Food Chem 2007; 55(4): 12801288.

28.- Legnani C. Frascaro M. Guazzaloca G. Ludovici S. Cesarano G.Coccheri S. Effects of a dried garlic preparation on fibrinolysis and platelet aggregation in healthy subjects.

Arzneimittelforschung 1993; 43(2): 119-122.

29.- $\quad$ Ali M. Bordia T.Mustafa T. Effect of raw versus boiled aqueous extract of garlic and onion on platelet aggregation. Prostaglandins Leukot Essent Fatty Acids 1999; 60(1): 43-47.

30.- Augusti K.T. Therapeutic and medicinal values of onions and garlic. In Onions and allied crops ed. Rabinowitch H. D. Brewster, J. L., Boca Raton, FL: CRC Press. 1990; 3: 94-108.

31.- Lawson L.D. Ransom D.K. Hughes B.G. Inhibition of whole blood platelet-aggregation by compounds in garlic clove extracts and commercial garlic products. Thromb Res 1992; 65(2): 141156.

32.- Lancaster J. Collin H. Presence of alliinase in isolated vacuoles and of alkyl cysteine sulphoxides in the cytoplasm of bulbs of onion (Allium cepa). Plant Sci. Lett 1981;22: 169-176. 33.- $\quad$ Banerjee S.K. Maulik S.K. Effect of garlic on cardiovascular disorders: a review. Nutr J 2002; $1: 4$.

34.- Block E. The organosulfur chemistry of the genus Allium. Implications for organic sulfur chemistry. Agnew. Chem., Int. Ed. Engl 1992; 31: 1135-1178.

35.- Block E. Naganathan S. Putman D. Zhao S.H. Allium chemistry: HPLC analysis of thiosulfinates from onion, garlic, wild garlic (ramsoms), leek, scallion, shallot, elephant 
(greatheaded) garlic, chive, Chinese chive. Uniquely high allyl to methyl ratios in some garlic samples. J. Agric. Food. Chem. 1992; 40: 2418-2430.

36.- $\quad$ Briggs W.H. Xiao H. Parkin K.L. Shen C. Goldman I.L. Differential inhibition of human platelet aggregation by selected Allium thiosulfinates. J Agric Food Chem 2000; 48(11): 5731-5735. 37.- Chen J.H. Chen H.I. Tsai S.J. Jen C.J. Chronic consumption of raw but not boiled Welsh onion juice inhibits rat platelet function. J Nutr 2000; 130(1): 34-37.

38.- $\quad$ Chen J. H. Chen H. I. Wang J. S. Tsai S. J.Jen C. J. Effects of Welsh onion extracts on human platelet function in vitro. Life Sci 2000; 66(17): 1571-1579.

39.- Dutta-Roy A.K. Crosbie L. Gordon M.J. Effects of tomato extract on human platelet aggregation in vitro. Platelets 2001; 12(4): 218-227.

40.- Yamamoto J. Taka T. Yamada K. Ijiri Y. Murakami M. Hirata Y. Naemura A. Hashimoto M. Yamashita T. Oiwa K. Seki J. Suganuma H. Inakuma T. Yoshida T. Tomatoes have natural antithrombotic effects. Br J Nutr 2003; 90(6): 1031-1038.

41.- O'Kennedy N. Crosbie L. Whelan S. Luther V. Horgan G. Broom J. Webb, D. Duttaroy A.K. Effects of tomato extract on platelet function: a double-blinded crossover study in healthy humans. Am J Clin Nutr 2006; 84(3): 561-569.

42.- O'Kennedy N. Crosbie L. van Lieshout M. Broom J. Webb D. Duttaroy A.K. Effects of antiplatelet components of tomato extract on platelet func tion in vitro and ex vivo: a time-course cannulation study in healthy humans. Am J Clin Nutr 2006; 84(3): 570-579.

43.- $\quad$ Arab L. Steck S. Lycopene and cardiovascular disease. Am J Clin Nutr 2000; 71(6 Suppl): 1691S-1695S; discussion 1696S-1697S.

44.- Altman R. Rouvier J.Weisenberger H. Identification of platelet inhibitor present in the melon (Cucurbitacea cucumis melo). Thromb Haemost 1985; 53(3): 312-313.

45.- Hawkey C. Howell M. Orally Induced Fibrinolysis. a Critical Assessment of Pineapple Protease. Thromb Diath Haemorrh 1964; 12: 382-390.

46.- Goldman I.L. Schwartz B.S. Kopelberg M. Kopelberg M. Debaene J.E. Schwartz B.S.

Antiplatelet activity in onion (Allium cepa) is sulfur dependent. Thromb Haemost 1996; 76(3): 450452.

47.- Kim G.N. Lee M.K. Park I. Acid and heat stability of the anticoagulative activity of an onion extract. Biosci Biotechnol Biochem 2002; 66(4): 859-861.

48.- Yamada K. Naemura A. Sawashita N. Noguchi Y. Yamamoto J. An onion variety has natural antithrombotic effect as assessed by thrombosis/thrombolysis models in rodents. Thromb Res 2004; 114(3): 213-220.

49.- Lucas J.S. Lewis S.A. Hourihane J.O. Kiwi fruit allergy: a review. Pediatr Allergy Immunol $2003 ; 14(6): 420-428$.

50.- $\quad$ Wills R.B.H. Lim J.S.K. Greenfield H. Composition of Australian foods. 31. Tropical and sub-tropical fruit. Food Technology in Australia 1986; 38(3): 118-123.

51.- Astrup T. Mullertz S. The fibrin plate method for estimating fibrinolytic activity. Arch Biochem Biophys 1952; 40(2): 346-351.

52.- Jung K.A. Song T.C. Han D. Kim I.H. Kim Y.E. Lee C.H. Cardiovascular protective properties of kiwifruit extracts in vitro. Biol Pharm Bull 2005; 28(9): 1782-1785.

53.- Pirotta F. de Giuli-Morghen C. Bromelain: anti-inflammatory and serum fibronolytic activity after oral administration in the rat. Drugs Exptl Clin Res 1978; 4: 1-20.

54.- Taussig S.J. Batkin S. Bromelain, the enzyme complex of pineapple (Ananas comosus) and its clinical application. An update. J Ethnopharmacol 1988; 22(2): 191-203.

55.- De-Giuli M. Pirotta F. Bromelain: interaction with some protease inhibitors and rabbit specific antiserum. Drugs ExpClin Res 1978; 4: 21-23.

56.- $\quad$ Fukao H. Yoshida H. Tazawa Y. Hada T. Antithrombotic effects of odorless garlic powder both in vitro and in vivo. Biosci Biotechnol Biochem 2007; 71(1): 84-90. 
57.- $\quad$ Bhushan S. Sharma S.P. Singh S.P. Agrawal S. Indrayan A. Seth P. . Effect of garlic on normal blood cholesterol level. Indian J Physiol Pharmacol 1979; 23(3): 211-214.

58.- Gadkari J.V. Joshi V.D. Effect of ingestion of raw garlic on serum cholesterol level, clotting time and fibrinolytic activity in normal subjects. J Postgrad Med 1991; 37(3): 128-131.

59.- Menon I.S. Kendal R.Y. Dewar H.A. Newell D.J. Effect of onions on blood fibrinolytic activity. Br Med J 1968; 3(5614): 351-352.

60.- $\quad$ Suzuki Y. Kondo K. Ichise H. Tsukamoto Y. Urano T.Umemura K. Dietary supplementation with fermented soybeans suppresses intimal thickening. Nutrition 2003; 19(3): 261-264.

61.- Suzuki Y. Kondo K. Matsumoto Y. Zhao B. Q. Otsuguro K. Maeda T. Tsukamoto Y. Urano T.Umemura K. Dietary supplementation of fermented soybean, natto, suppresses intimal thickening and modulates the lysis of mural thrombi after endothelial injury in rat femoral artery. Life Sci 2003; 73(10): 1289-1298. 